$\Delta$ rkivoc

Free to Authors and Readers
A Platinum Open Access Journal

for Organic Chemistry
Review

Arkivoc 2021, part vii, 164-176

\title{
Hypervalent iodine reagents as activators in glycosylation reactions
}

\author{
Koji Morimoto, ${ }^{a, b *}$ Yasuyuki Kita ${ }^{b}$, and Tetsuya Kajimoto ${ }^{a, b *}$ \\ a College of Pharmaceutical Sciences, Ritsumeikan University, 1-1-1 Nojihigashi, Kusatsu, Shiga, 525-8577, \\ Japan \\ ${ }^{b}$ Research Organization of Science and Technology, Ritsumeikan University, 1-1-1 Nojihigashi, Kusatsu, Shiga, \\ 525-8577, Japan \\ Email: k-morimo@fc.ritsumei.ac.jp; kajimoto@fc.ritsumei.ac.jp
}

Received 09-05-2021

Accepted 11-19-2021

Published on line $12-16-2021$

\section{Abstract}

Glycoconjugates are present in various bioactive natural products and medicianlly important compounds; therefore, the development of novel and efficient glycosylation reactions is an important objective in organic synthesis. Thioglycosides are glycosyl donors that can act as protected glycosides at the anomeric position and can be selectively activated by the appropriate reagents under mild conditions. Although numerous methods employing oxidizing agents as an activator of the thioglycoside are currently available, more versatile approaches are still required to attain higher chemical yield and anomer selectivities. We have found that trivalent hypervalent iodine reagents, such as phenyliodine diacetate (PIDA) and phenyliodine bis(trifluoroacetate) (PIFA), display a much greater reactivity than heavy metal oxidizers or monovalent iodonium species to activate the thioglycosides. We now report novel $O$ - and $\mathrm{N}$-glycosylation reactions of the thioglycosides, activated by a combination of the hypervalent iodine reagent and Lewis acid.

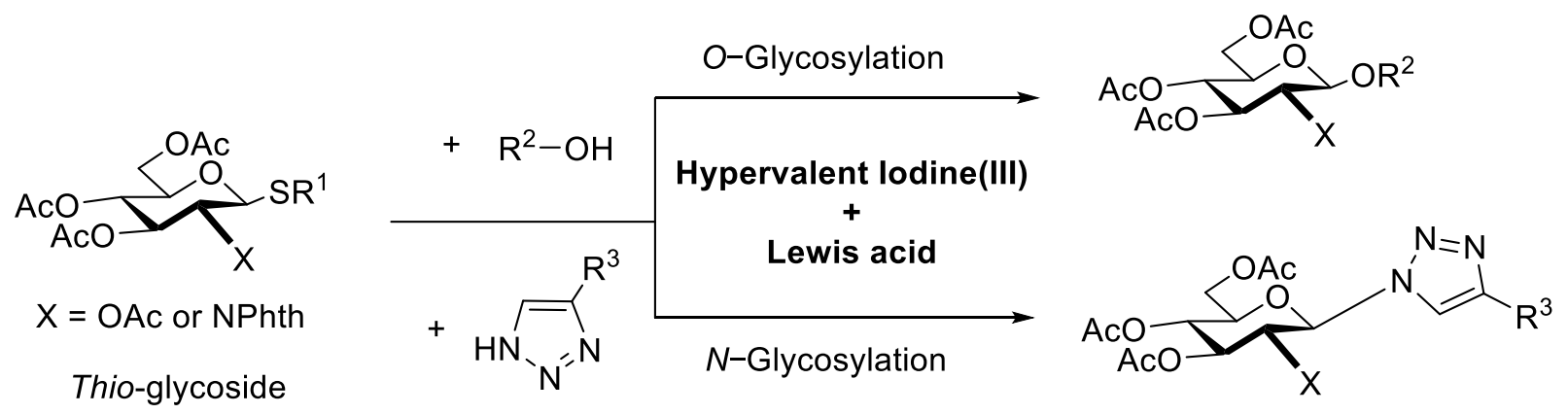

Keywords: Glycosylation, thioglycoside, hypervalent iodine, metal-free 


\section{Table of Contents}

1. Introduction

2. Glycosylation of Thioglycosides as Sugar Donors using Hypervalent lodine Reagents

3. Extension to Disaccharide Synthesis

4. Development of Glycosylation Reactions using Thioglycosides derived from Odorless Thiols

5. N-Glycosylation of Thioglycosides

6. Conclusions

References

\section{Introduction}

The development of glycosylation reactions for the efficient synthesis of naturally-occurring or synthesis bioactive glycosides is an important topic in organic chemistry. For the glycosylation reaction, glycosyl donors are activated by various activators to react with glycosyl acceptors to generate a glycosidic bond as well as a new asymmetric center at the anomeric carbon. Thus, an efficient glycoside synthesis should be precisely controlled not only for forming either an $\alpha$ or $\beta$ glycosidic linkage but also for reacting with some of the hydroxyl or amino groups of the acceptor substrates. A number of glycosylation methods has been developed based on variations in the activating groups of the glycosyl donor, nucleophilic nature of the hydroxyl group of the acceptor, kinds of activators, and solvent. ${ }^{1,2}$ However, the development of new and efficient glycosylation reactions is still desired to attain higher chemical yields and anomer selectivities. The Schmidt method using glycosyl chloroimidates and the Suzuki method using glycosyl fluorides have been estimated to be excellent methods. However, recently, thioglycosides have been widely employed as the glycosyl donor among the many kinds of donor substrates. In general, the sulfide bonds are unaffected under the conditions for the protection or deprotection of hydroxyl and amino groups, thus can behave as a protecting group of the anomeric position. Meanwhile, the C-S bond can be specifically activated using appropriate reagents. The thioglycoside activation methods reported to date can be classified into four main categories based on the reagents used: i.e., (1) metal oxidizers, (2) halonium reagents, (3) organosulfur reagents, and (4) singleelectron transfer (SET) reagents (Scheme 1).

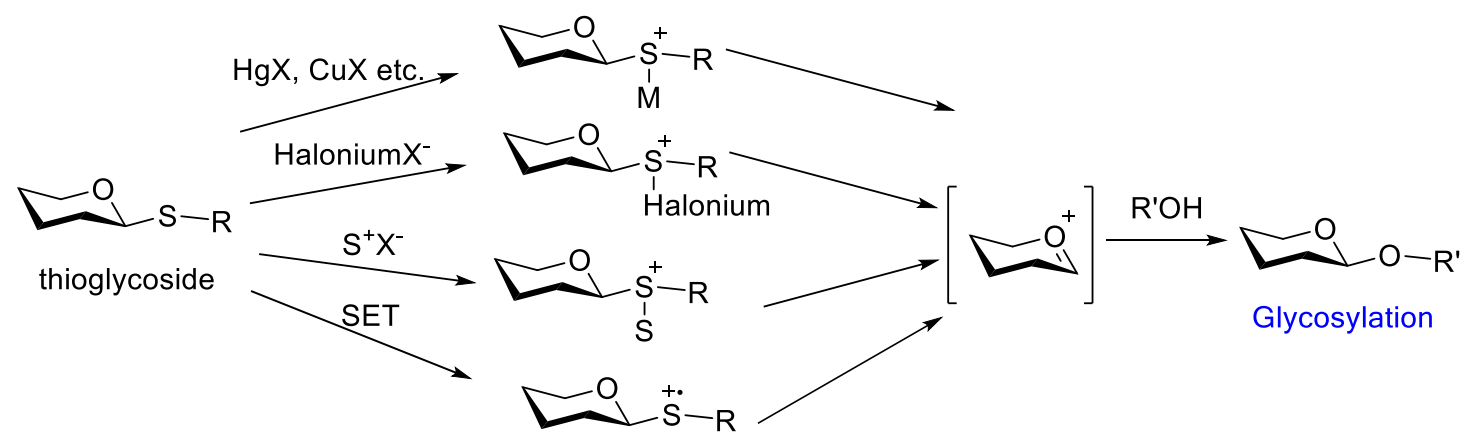

thionium cationic species

Scheme 1. Thioglycoside activation methods for glycosylation. 
All of the above-listed activators produce thionium cationic species, which act as intermediates in the glycosylation reaction. Classical methods entailing the use of heavy metal salts as activators, such as mercury and lead ions, were often employed, despite being highly unfavorable in terms of the impetus for green chemistry. ${ }^{3-6}$ Van Boom and his colleagues reported the use of a stoichiometric amount of $\mathrm{N}$-iodosuccinimide (NIS) as the halonium species and a catalytic amount of trifluoromethanesulfonic acid (TfOH) as the promoter. ${ }^{7,8}$ This method is less toxic than typical protocols that use heavy metal oxidizers and is widely adopted in natural product synthesis. Despite its usefulness, NIS has a disadvantage that it has no modifiable groups in the structure such as an aromatic ring. Structural modification of the reagents could control the reactivity in case the reaction yield is poor. Thus, we describe in the present article, our recent development for the glycosylation reaction of thioglycosides using hypervalent iodine(III) reagents, of which the structure can be modified by replacing substituents on the aromatic ring or ligands on iodine(III). The most important advantage of this protocol is the use of environmentally-benign metal-free conditions.

\section{Glycosylation of Thioglycosides as Sugar Donors using Hypervalent lodine Reagents}

Since the 1990s, green chemistry has become increasingly relevant. In this regard, low-toxic and safe hypervalent iodine reagents have been attracting attention as alternatives to heavy metal oxidizers. To date, we have developed several synthetic reactions using hypervalent iodine reagents and applied them to the total syntheses of bioactive natural products. We have developed a simple method for the synthesis of heterocyclic compounds by applying this methodology to intramolecular ring-closing reactions. ${ }^{9-14}$ Kita, one of the authors, also developed a method for the introduction of azide groups at the $\alpha$-position of the sulfides, entailing sulfur oxidation via the formation of active iodonium intermediates using hypervalent iodine(III) reagents, thereby providing a new method for the synthesis of heterocyclic compounds. ${ }^{15,16}$ The total synthesis of the natural product, makaluvamine $F$, has been achieved using the developed methodology (Scheme 2). ${ }^{17,18}$

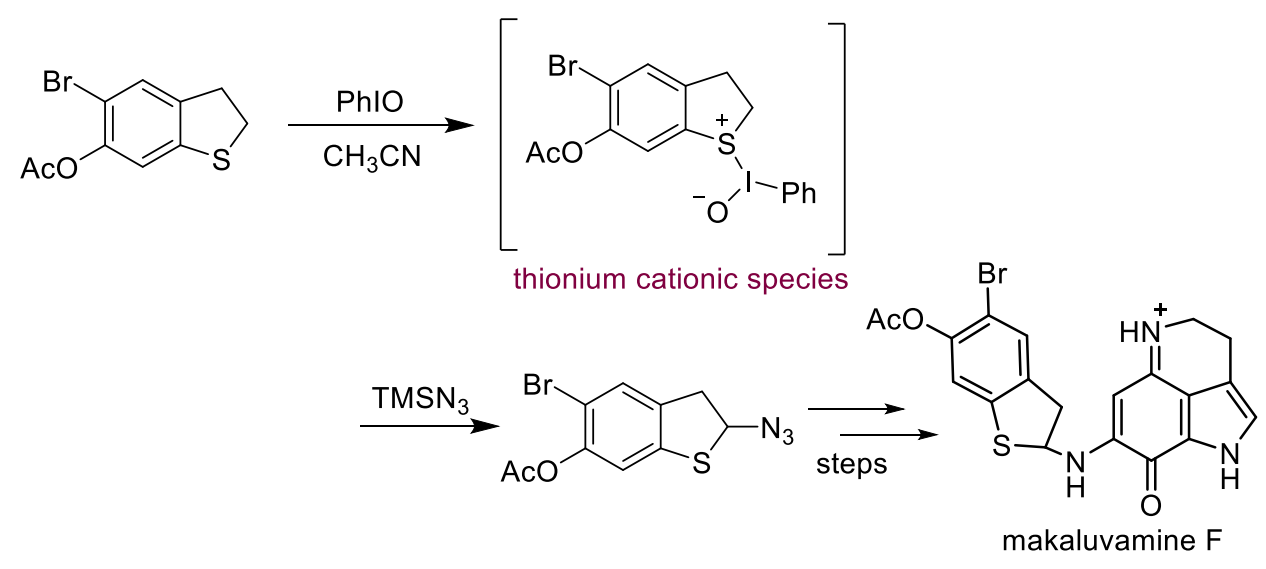

Scheme 2. The synthesis of makaluvamine F involving sulfur oxidation.

Subsequently, various sulfide oxidation methods using other reagents have been reported, but only a few reports have been published about the glycosylation of thioglycosides via activation by hypervalent iodine. ${ }^{19}$ Herein, a readily available thioglycoside, methyl 2,3,4,6-tetra-O-acetyl-1-thio- $\beta$-D-glucopyranoside $1 \mathrm{a}$, was used as a glycosyl donor, and methyl 2,3,4-tri-O-benzyl- $\alpha$-D-glucopyranoside $\mathbf{2}$ was used as the acceptor, and 
the reaction conditions were optimized (Table 1). Initially, the reaction was performed in $\mathrm{CH}_{2} \mathrm{Cl}_{2} \mathrm{using}$ phenyliodine diacetate (PIDA) in an equimolar amount to $\mathbf{1 a}$ in the presence of trifluoromethanesulfonic acid (TfOH) at room temperature; however, the reaction did not proceed and the desired product was not obtained (Entry 1). Therefore, we conducted the reaction at $-78{ }^{\circ} \mathrm{C}$ under otherwise unchanged conditions and obtained the desired glycosylated product $3 a$ in $20 \%$ yield (Entry 2). Next, phenyl iodinebis(trifluoroacetate) (PIFA) was used as the iodine reagent in combination with $\mathrm{TfOH}$ to obtain product $\mathbf{3 a}$ in $40 \%$ yield (Entry 3 ). However, when the reaction was carried out using PhI(OH)OTs, the desired product was not produced (Entry 4). Furthermore, we attempted to use trimethylsilyl trifluoromethanesulfonic acid (TMSOTf) as a Lewis acid, but the yield was not improved by changing the additive (Entry 5). Although the role of TfOH is unclear, it is suggested that it coordinates to the carbonyl oxygen of the trifluoroacetate of PIFA to reduce the electron density of iodine and that this function plays an important role in the activation of thioglycosides and the formation of cationic intermediates. The reaction of alcohol $\mathbf{2}$ with the cationic intermediate is considered to have produced the glycosylated product 3a. Interestingly, glycosylation using methyl 2-deoxy-2-phthalimido3,4,6-tri-O-acetyl-1-thio- $\beta$-D-glucopyranoside $\mathbf{1 b}$ as a glycosyl donor instead of $\mathbf{1 a}$ provided the desired product $\mathbf{3 b}$ in $83 \%$ yield (Entry 6 ). Thioglycoside containing a phthalimide group at the $\mathrm{C}-2$ position of the pyranose ring was found to be a more useful glycosyl donor for the glycosylation reaction.

Table 1. Optimization of hypervalent-iodine(III)-mediated glycosylation reaction conditions

\begin{tabular}{|c|c|c|c|c|c|}
\hline $\begin{array}{l}1 \mathbf{a}: \mathrm{R}=\mathrm{OAc} \\
\mathbf{b}: \mathrm{R}=\mathrm{NPhth}\end{array}$ & 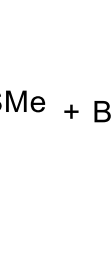 & OMe & $\begin{array}{l}\text { ( } 1 \text { equiv.) } \\
\text { equiv.) } \\
\text { S4A }\end{array}$ & AcO & \\
\hline Entry & Donor & I (III) & Additive & Temp $\left({ }^{\circ} \mathrm{C}\right)$ & Yield (\%) \\
\hline 1 & $1 a$ & PIDA & TfOH & r.t. & trace \\
\hline 2 & $1 a$ & // & $\mathrm{TfOH}$ & -78 & 20 \\
\hline 3 & $1 a$ & PIFA & $\mathrm{TfOH}$ & $\mathrm{TfOH}$ & 40 \\
\hline 4 & $1 a$ & $\mathrm{Phl}(\mathrm{OH}) \mathrm{OTs}$ & $\mathrm{TfOH}$ & $\mathrm{TfOH}$ & trace \\
\hline 5 & $1 a$ & PIFA & TMSOTf & $\mathrm{TfOH}$ & 35 \\
\hline 6 & $1 b$ & // & $\mathrm{TfOH}$ & $\mathrm{TfOH}$ & 83 \\
\hline
\end{tabular}

Next, we examined the substrate scope for this glycosylation reaction employing the optimized conditions shown below (Scheme 3). It was found that the glycosylation reaction smoothly proceeded at room temperature when various alcohols were used as sugar acceptors, and the corresponding products were obtained in good yields. The reaction also proceeded with the use of secondary alcohols, such as (-)-menthol, $\beta$-cholestanol, fenchol, and 2-adamantanol, and the corresponding glycosylated compounds 3c-3f were obtained in good yields. Furthermore, a decrease in the yield was not observed when 1-adamantanol, a bulky tertiary alcohol, was used, affording the desired glycosylated product $\mathbf{3 g}$. The reaction smoothly proceeded when (+)- and (-)-borneol were used, and the corresponding products $\mathbf{3 h}$ and $\mathbf{3 i}$ were obtained in good yields. In this case, the reaction $\beta$-selectively proceeded due to the involvement of the adjacent phthalimide groups. 


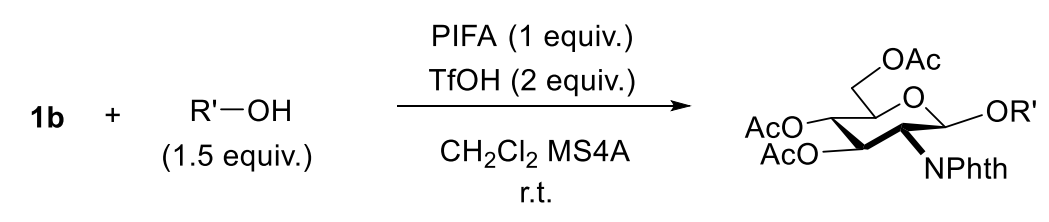

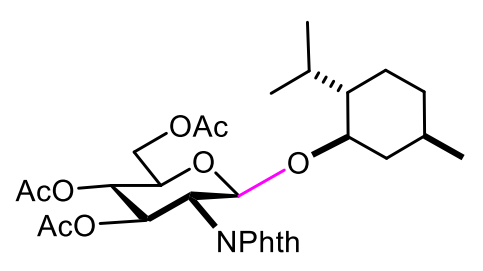

3c $57 \%$

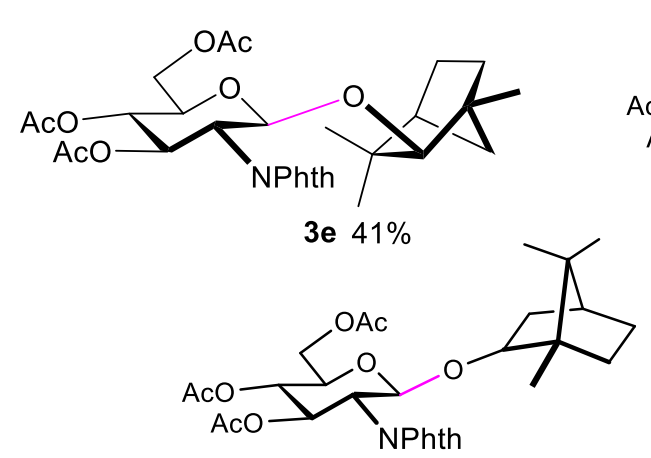

3h $63 \%$

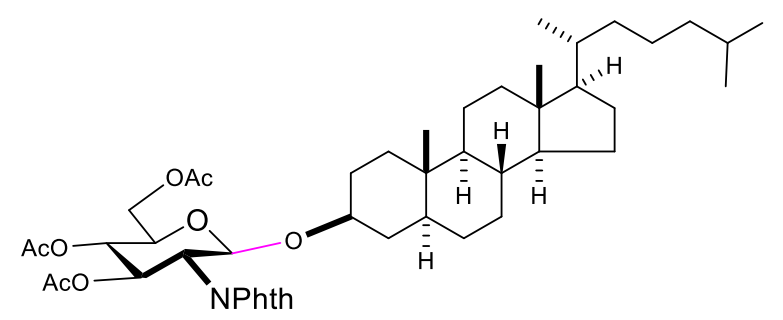

3d $60 \%$

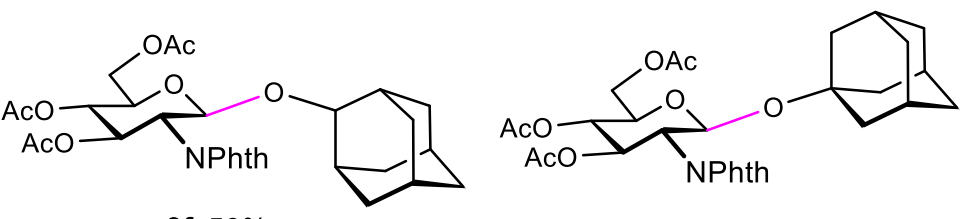

3f $58 \%$

3g $58 \%$

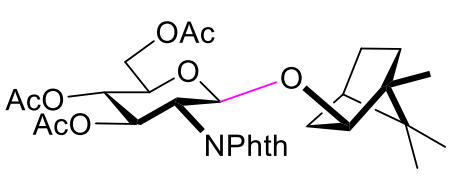

3i $73 \%$

Scheme 3. Glycosylation substrate scope.

\section{Extension to Disaccharide Synthesis}

Having achieved glycosylation of the methyl thioglycosides (1a, 1b) with various alcohols using hypervalent iodine(III), we embarked on its application for oligosaccharide synthesis. For the synthesis of oligosaccharides, the key step is attributed to the selective and high-yielding formation of the glycosidic linkages. Our glycosylation protocol, in which the thioglycosides are used as sugar donors in the presence of PIFA/TfOH as an activator, can avoid the by-production of anomeric isomers and seemed to allow the oligosaccharide synthesis in good yields. Thus, we initially applied our hypervalent-iodine-mediated glycosylation reaction to the disaccharide synthesis.

Under the standard reaction conditions, the corresponding glycosylated compounds were obtained in good yield (Scheme 4). In this case, conducting the reaction at $-78{ }^{\circ} \mathrm{C}$ led to an improvement in the yield. When thioglycoside 1a and the benzoyl-protected acceptor bearing a hydroxyl group at the 6-position were used, the desired glycosylated product $\mathbf{3} \mathbf{j}$ was obtained in high yield. Similarly, the use of an acetal-protected acceptor gave the desired product $\mathbf{3 k}$ in good yield. Gratifyingly, the glycosylation of galactose at the 4position gave the corresponding product $\mathbf{3 l}$ in $55 \%$ yield. Glycoprotein glycans are a remarkably diverse group of molecules, classified according to their binding mode to proteins, with particularly structurally complex glycans often expressing glycoconjugates important for glycosylation at their terminus, such as galactose. However, glycosylation reactions at the axial hydroxyl group at the 4-position of galactose are rare, and generally low-yielding; therefore, obtaining the glycosylated galactose product in $55 \%$ yield is a notable 
achievement, demonstrating the usefulness of this reaction. The reaction generally provided moderate yields, although the yields were lower when the reaction was performed with a donor, confirming that products $3 \mathrm{~m}$ and $\mathbf{3 n}$ were obtained in a $\beta$-selective manner. Moreover, it was found that when a galactose derivative was used as the sugar donor, favourable reactivity was maintained and glycosyl product 30 was obtained in $63 \%$ yield. ${ }^{20}$ This is an example of a reaction between a glycosyl sugar donor with an electron-withdrawing group, and a glycol-acceptor with an electron-donating group, which is generally considered challenging. ${ }^{21}$

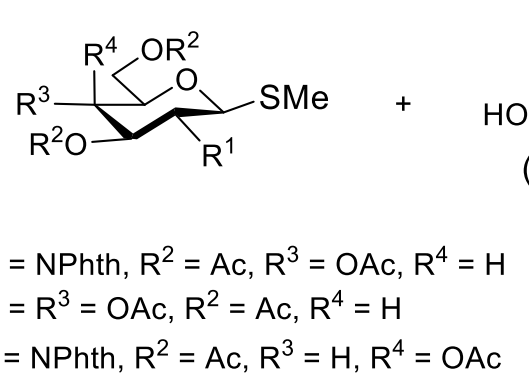

$$
\begin{aligned}
& \text { 1b }: R^{1}=\text { NPhth, } R^{2}=A c, R^{3}=O A c, R^{4}=H \\
& \text { 1c }: R^{1}=R^{3}=O A c, R^{2}=A c, R^{4}=H \\
& \text { 1d }: R^{1}=\text { NPhth, } R^{2}=A c, R^{3}=H, R^{4}=\text { OAc }
\end{aligned}
$$
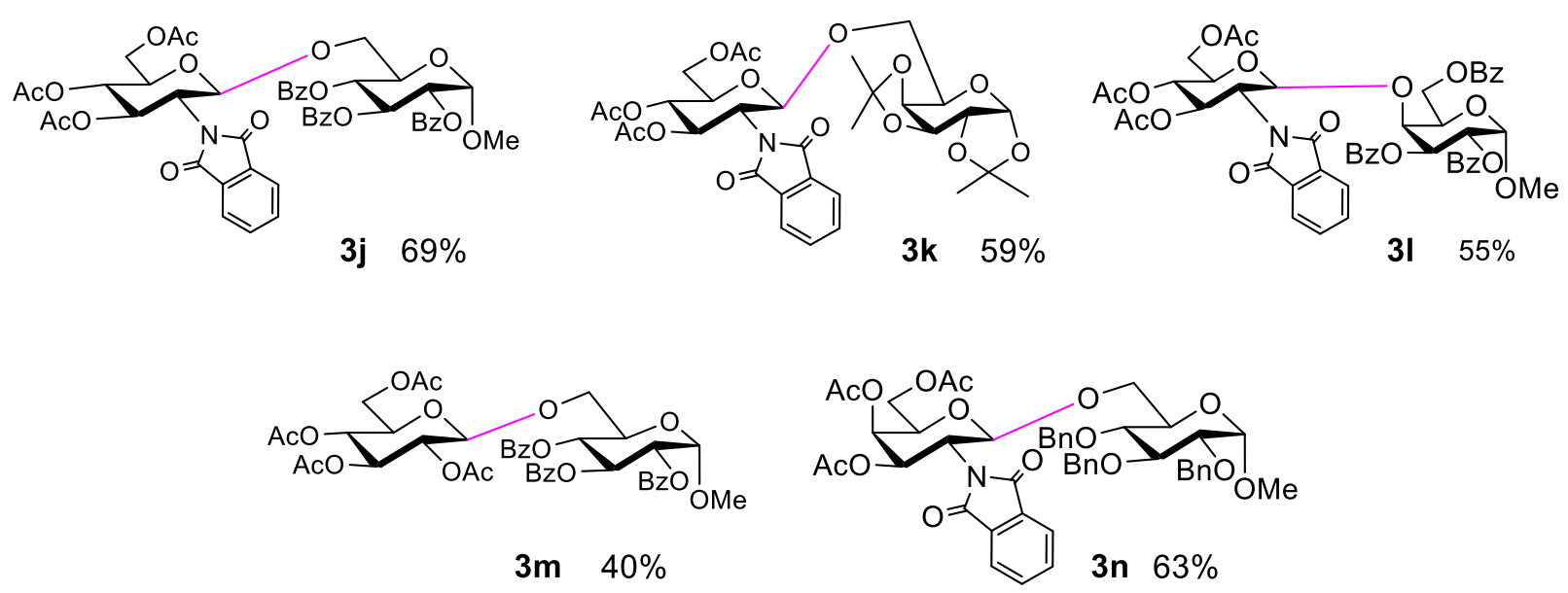

Scheme 4. Scope of glycosylation for disaccharide synthesis.

\section{Development of Glycosylation Reactions using Thioglycosides Derived from Odorless} Thiols

Next, we turned our attention to optimizing the mercaptane moiety of the thioglycoside. Methyl trimethylsilyl sulfide and benzenethiol are commonly used for the preparation of the appropriate thioglycosides; however, paying attention to the working environment is important since an unpleasant odor is generated during preparation of the thioglycosides and glycosylation reactions. Kajimoto and his colleagues developed $p$ dodecylbenzenethiol and $p$-octyloxybenzenethiol as odorless alternatives to benzenethiol. We then employed the odorless thioglycoside $1 \mathbf{e}$ as the donor substrate for our glycosylation reaction. ${ }^{21-23}$

The results are summarized in Scheme 5. The reaction conditions and the acceptor substrates were the same as those in Scheme 3. The reaction with the glycosyl donor 1e afforded the corresponding products in higher yields than for the reaction with $\mathbf{1 b} .^{24}$ 

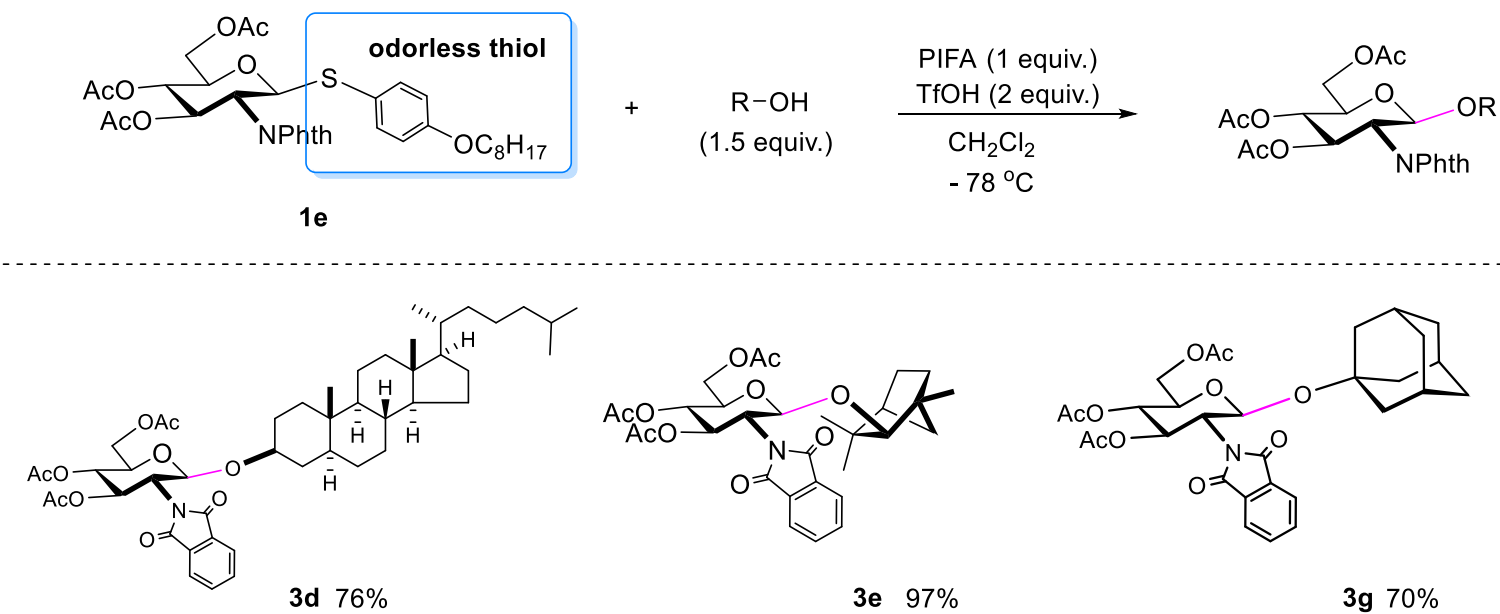

3d $76 \%$

3e $97 \%$

3g $70 \%$

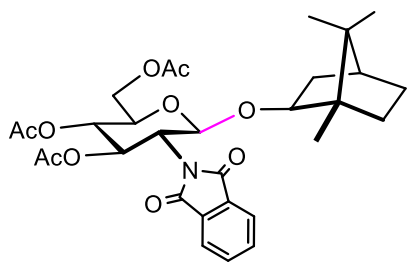

3h $93 \%$

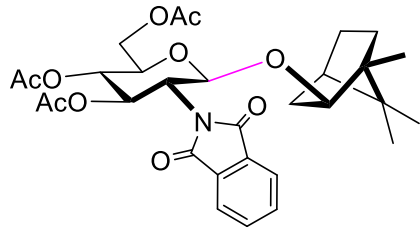

3i $84 \%$

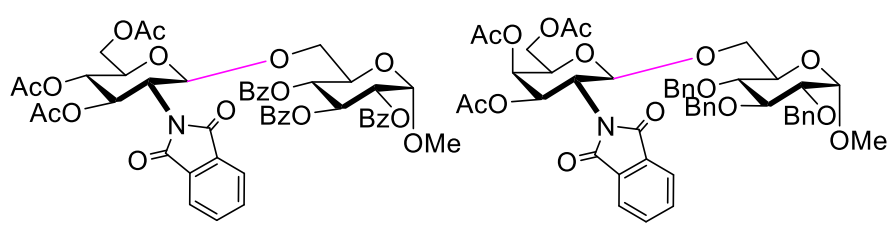

3j $87 \%$

3n $63 \%$

Scheme 5. Scope of glycosylation using thioglycosides derived from odorless $p$-octyloxybenzenethiol.

A trisaccharide synthesis was also challenged using a disaccharide 4 as the donor and a benzylated glycoside $\mathbf{2}$ as the acceptor substrate (Scheme 6). The reaction smoothly proceeded providing the desired trisaccharide $\mathbf{5}$ in high yield (82\%). ${ }^{25}$ Although various methods for the synthesis of the oligosaccharides have been reported to date, most of them, except for the Danishefsky method, depend on the glycosylation starting from the reducing end. ${ }^{26}$ The general strategy is sometimes not suitable for use in the final stages of the total synthesis due to their limited substrate scope and low yields. We have now confirmed that the present method could provide another synthetic strategy for oligosaccharide synthesis because the glycosylation in Scheme 6 proceeds from the non-reducing end.
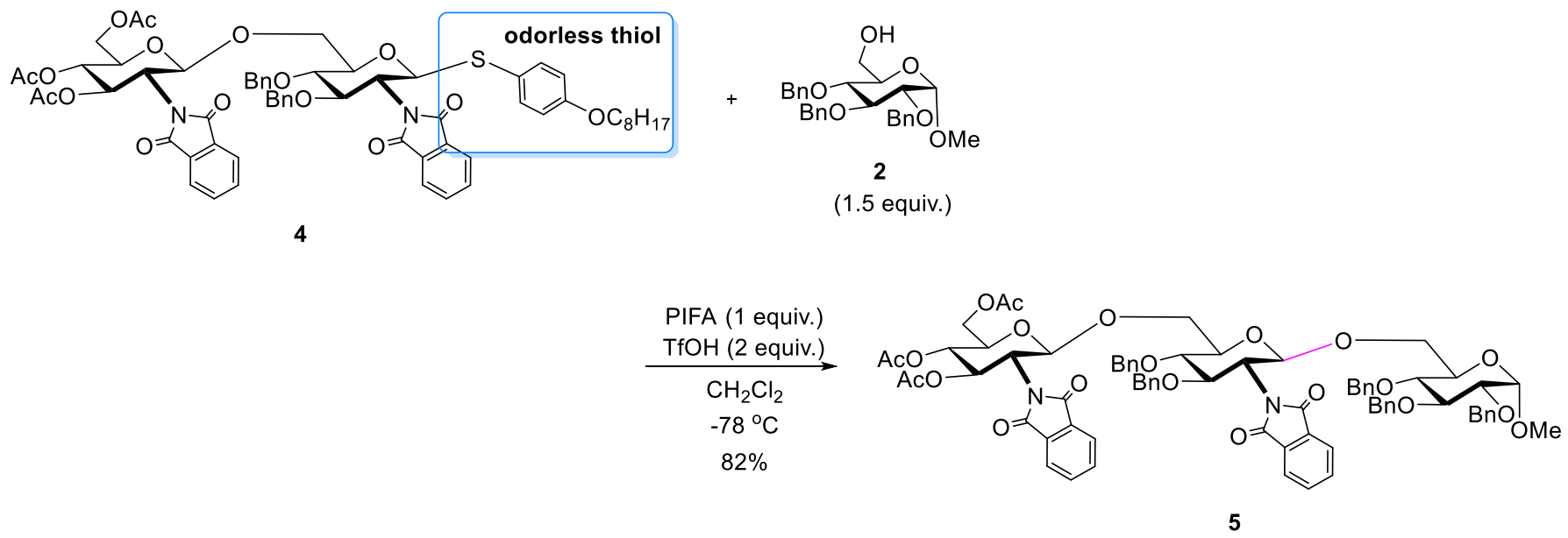

Scheme 6. Trisaccharide synthesis from thioglycosides derived from odorless $p$-octyloxybenzenethiol. 


\section{N-Glycosylation of Thioglycosides}

Since the glycosyl derivatives with carbon-nitrogen (C-N) bonds at the anomeric position are known to play an important role in a variety of biological events, ${ }^{27-29}$ the development of useful $\mathrm{N}$-glycosylation reactions is an important endeavor. However, the $\mathrm{N}$-glycosylation methods are relatively underdeveloped compared to the $\mathrm{O}$-glycosylation reactions. Among the $\mathrm{N}$-glycans, the glycosylazoles do not only show a potent inhibitory activity against the glycosidases and glycosyltransferases, but are also attractive molecules used as biologically-inactive linkers. Thus, the development of efficient methods for accessing these glycosyl azoles has received considerable attention. Various types of $\mathrm{N}$-bonded glycosyl triazoles have been reported, most of which are generally synthesized via the Huisgen [3 + 2] cycloaddition; however, due to the use of an unstable monovalent $\mathrm{Cu}$ catalyst and its persistent property, a tedious procedure to remove the $\mathrm{Cu}$ residue after the reaction is necessary. ${ }^{30,31}$

As mentioned above, we have recently developed the glycosylation of thioglycosides using hypervalent iodine(III) reactants as activators. Thus, a simple method for the synthesis of glycosyl azoles using hypervalent iodine reagents as activators was investigated. First, the glycosylation of thioglycoside $\mathbf{1 b}$ with 1,2,3benzotriazole 6a was investigated. However, product 7a was obtained in only moderate yield when PIFA was used in the presence of $\mathrm{TfOH}$, which had given good results in our previous glycosylation reactions. Therefore the use of additives was investigated, and $\mathrm{N}$-glycosylated 7 a was obtained in good yield when the reaction was conducted at $80^{\circ} \mathrm{C}$ in dichloroethane using TMSOTf as the Lewis acid (Scheme 7).

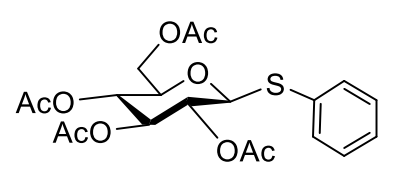

$1 \mathbf{f}$

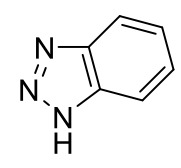

$6 a$

(1.5 equiv.)

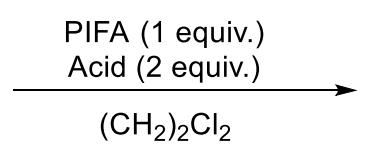

$\left(\mathrm{CH}_{2}\right)_{2} \mathrm{Cl}_{2}$

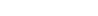




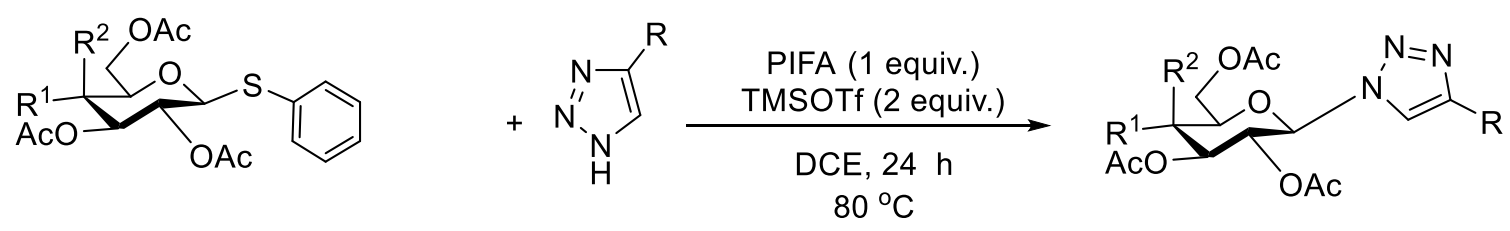

$$
\begin{aligned}
& \text { 1f }: R^{1}=O A c, R^{2}=H \\
& \text { 1g: } R^{1}=H, R^{2}=O A C
\end{aligned}
$$

6b-h

(2.0 equiv.)

7b-f

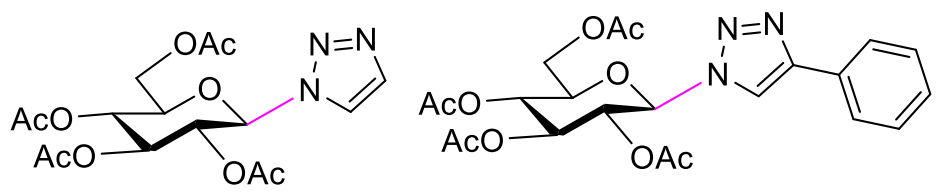

7b $47 \%$

7c $51 \%$

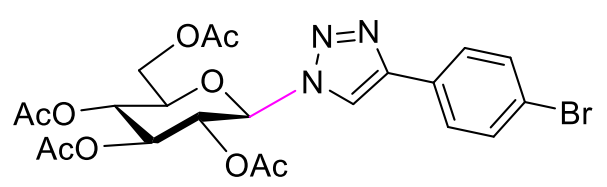

7 e $54 \%$

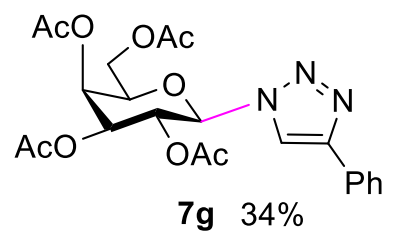

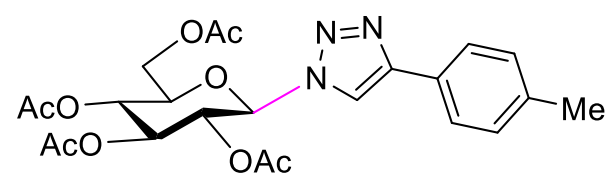

7d $53 \%$

7f $41 \%$

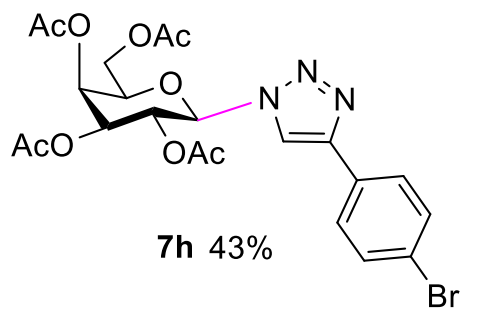

Scheme 8. Scope of N-glycosylation.

Recently, this reaction has been extended to the practical N-glycosylation of thioglycoside $\mathbf{1 h}$ derived from the odorless $p$-octyloxybenzenethiol. ${ }^{32}$

To demonstrate the practicality and efficiency of the developed method, scaled-up reactions were performed. It was possible to synthesize the $\mathrm{N}$-glycosylated product $7 \mathrm{a}$ on a gram scale $(2 \mathrm{mmol})$ from the reaction of the thioglycoside $\mathbf{1 h}$ obtained from the odorless $p$-octyloxybenzenethiol and benzotriazole $6 \mathbf{a}$ (Scheme 9).

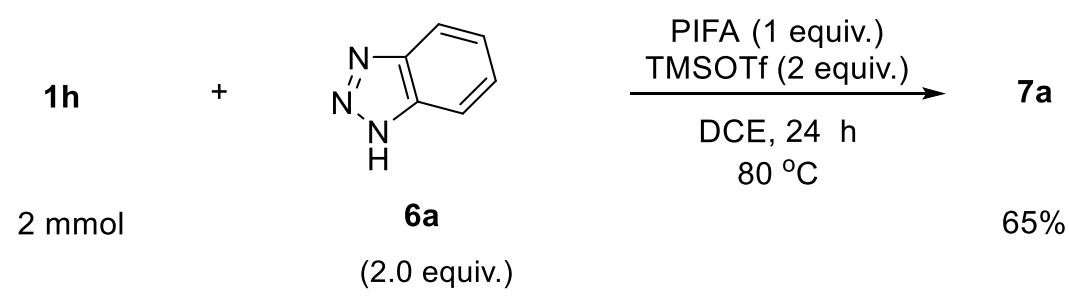

Scheme 9. Large scale synthesis of 7a. 


\section{Conclusions}

Metal-free glycosylation reactions using hypervalent iodine reagents, which are environmentally more benign reagents than heavy metal ions, were successfully developed. Using the present method, disaccharides can be easily synthesized then a three connected glycan can be generated by repeating the same reaction. Hopefully, this developed glycosylation method, in which environmentally benign organoiodine(III) reagents and odorless p-octyloxybenzenethiol were used, will contribute to the establishment of clean manufacturing technologies in the glycosciences. It is expected that the efficient synthesis of cyclic peptides, oligosaccharides/nucleic acids, glycopeptides, and other compounds will contribute to the development of life science research.

\section{Acknowledgements}

This work was partially supported by Grants-in-Aid for Scientific Research (A) (JSPS KAKENHI Grant Number 24249001) from the Japan Society for the Promotion of Science (JSPS), a Grant-in-Aid for Scientific Research on Innovative Areas 'Advanced Molecular Transformation by Organocatalysts' (MEXT Grant Number 23105006) from The Ministry of Education, Culture, Sports, Science and Technology (MEXT), and the Ritsumeikan Global Innovation Research Organization (R-GIRO) project. K.M. acknowledges support from the Grant-in-Aid for Scientific Research (C) Grant Number 20 K05520.

\section{References}

1. Codée, J. D. C.; Litjens, R. E. J. N.; van den Bos, L. J.; Overkleeft, H. S.; van der Marel, G. A. Chem. Soc. Rev. 2005, 34, 769. https://doi.org/10.1039/b417138c

2. Lian, G.; Zhang, X.; Yu, B. Carbohydr. Res. 2015, 403, 13. https://doi.org/10.1016/j.carres.2014.06.009

3. Ferrier, R. J.; Hay, R. W.; Vethaviyasar, N. Carbohydr. Res. 1973, 27, 55. https://doi.org/10.1016/S0008-6215(00)82424-6

4. Van Cleve, J. W. Carbohydr. Res. 1979, 70, 161. https://doi.org/10.1515/kant.1979.70.1-4.149

5. Garegg, P. J.; Henrichson, C.; Norberg, T. Carbohydr. Res. 1983, 116, 162. https://doi.org/10.1016/S0008-6215(00)90965-0

6. Sato, S.; Mori, M.; Ito, Y.; Ogawa, T. Carbohydr. Res. 1986, 155, C6. https://doi.org/10.1016/S0008-6215(00)90163-0

7. Konradsson, P.; Udodong, U. E.; Fraser-Reid, B. Tetrahedron Lett. 1990, 31, 4313. https://doi.org/10.1016/S0040-4039(00)97609-3

8. Sliedregt, L. A. J. M.; van der Marel, G. A.; van Boom, J. H. Tetrahedron Lett. 1994, 35, 4015. https://doi.org/10.1016/S0040-4039(00)76728-1

9. Kita, Y.; Tohma, H.; Yakura, T. Trends Org. Chem. 1992, 3, 113.

10. Tohma, H.; Kita, Y. in Topics in Current Chemistry, 2003, pp. 209-248. https://doi.org/10.1007/3-540-46114-0 8

11. Tohma, H.; Kita, Y. Adv. Synth. Catal. 2004, 346, 111. 
https://doi.org/10.1002/adsc.200303203

12. Kita, Y.; Dohi, T. Chem. Rec. 2015, 15, 886.

https://doi.org/10.1002/tcr.201500020

13. Dohi, T.; Kita, Y. Top. Curr. Chem. 2016, 373, 1.

14. Morimoto, K.; Dohi, T.; Kita, Y. Synlett 2017, 28, 1680.

https://doi.org/10.1055/s-0036-1588455

15. Matcha, K.; Narayan, R.; Antonchick, A. P. Angew. Chem., Int. Ed. 2013, 52, 7985.

https://doi.org/10.1002/anie.201303550

16. Wu, Z.; Xu, P.; Zhou, N.; Duan, Y.; Zhang, M.; Zhu, C. Chem. Commun. 2017, 53, 1045. https://doi.org/10.1039/C6CC08779E

17. Kita, Y.; Egi, M.; Ohtsubo, M.; Saiki, T.; Takada, T.; Tohma, H. Chem. Commun. 1996, 2225. https://doi.org/10.1039/cc9960002225

18. Kita, Y.; Egi, M.; Tohma, H. Chem. Commun. 1999, 143.

https://doi.org/10.1039/a808715f

19. Fukase, K.; Kinoshita, I.; Kanoh, T.; Nakai, Y.; Hasuoka, A.; Kusumoto, S. Tetrahedron 1996, $52,3897$. https://doi.org/10.1016/S0040-4020(96)00057-9

20. Kajimoto, T.; Morimoto, K.; Ogawa, R.; Dohi, T.; Kita, Y. Eur. J. Org. Chem. 2015, 2015, 2138. https://doi.org/10.1002/ejoc.201500186

21. Hasegawa, J.; Hamada, M.; Miyamoto, T.; Nishide, K.; Kajimoto, T.; Uenishi, J.; Node, M. Carbohydr. Res. 2005, 340, 2360.

https://doi.org/10.1016/i.carres.2005.07.011

22. Kajimoto, T.; Ishioka, Y.; Katoh, T.; Node, M. Bioorg. Med. Chem. Lett. 2006, 16, 5736. https://doi.org/10.1016/i.bmcl.2006.08.095

23. Kajimoto, T.; Ishioka, Y.; Katoh, T.; Node, M. J. Carbohydr. Chem. 2007, 26, 469. https://doi.org/10.1080/07328300701738027

24. Kajimoto, T.; Morimoto, K.; Ogawa, R.; Dohi, T.; Kita, Y. Chem. Pharm. Bull. 2016, 64, 838. https://doi.org/10.1248/cpb.c16-00203

25. Morimoto, K.; Yanase, K.; Odaka, I.; Kita, Y.; Kajimoto, T. Heterocycles 2019, 99, 680. https://doi.org/10.3987/COM-18-S(F)35

26. Seeberger, P. H.; Danishefsky, S. J. Acc. Chem. Rev. 1998, 31, 685. https://doi.org/10.1021/ar9600648

27. Taylor, M. E.; Drickamer, K. Introduction to Glycobiology, 2006.

28. Owens, N. W.; Schweizer, F. in Comprehensive Biotechnology, Second Edition, 2011, Vol. 1, pp. 122-138.

29. Parsons, T. B.; Struwe, W. B.; Gault, J.; Yamamoto, K.; Taylor, T. A.; Raj, R.; Wals, K.; Mohammed, S.;Robinson, C. V.; Benesch, J. L. P.; Davis, B. G. Angew. Chem. Int. Ed. 2016, 55, 2361. https://doi.org/10.1002/anie.201508723

30. Kolb, H. C.; Finn, M. G.; Sharpless, K. B. Angew. Chem. Int. Ed. 2001, 40, 2004. https://doi.org/10.1002/1521-3773(20010601)40:11<2004::AID-ANIE2004>3.0.CO;2-5

31. Mandoli, A. Molecules 2016, 21, 1174. https://doi.org/10.3390/molecules21091174

32. Morimoto, K.; Yanase, K.; Kamitanaka, T.; Kajimoto, T. Heterocycles 2021, 103, 809. https://doi.org/10.3987/COM-20-S(K)47 


\section{Authors' Biographies}

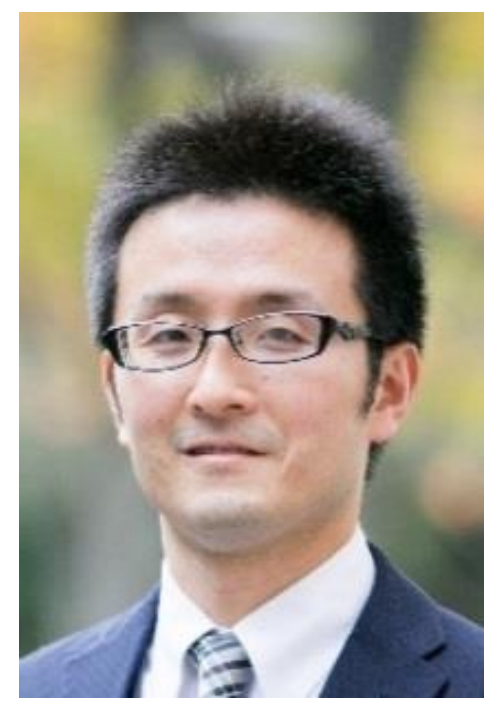

Koji Morimoto was born and grew up in Nara, Japan. He studied organic chemistry at the Graduate School of Pharmaceutical Sciences, Osaka University (2000-2009) where he obtained his Ph.D. from Osaka University. After obtaining his Ph.D., he worked as a researcher at Ritsumeikan University with Professor Yasuyuki Kita. In 2016, he got a position of assistant professor at Ritsumeikan University. He received the Award for Young Scientists at Kinki Branch Office (2010). In 2018 he also received a researcher's award from The Pharmaceutical Society of Japan Division of Organic Chemistry. The current research theme is the development of novel reactions in organic synthesis using hypervalent iodine reagents.

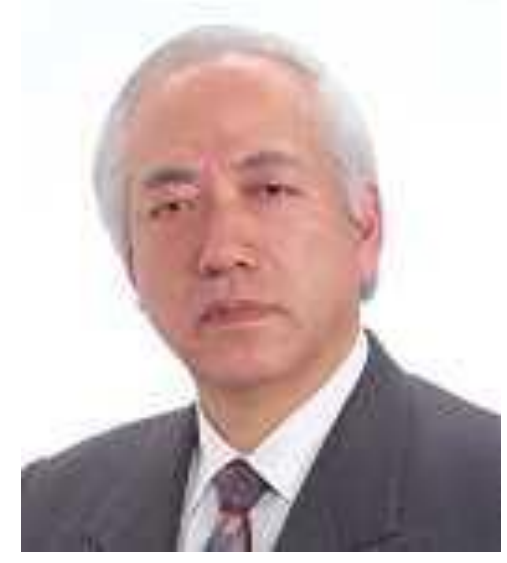

Yasuyuki Kita was born in 1945 in Osaka, Japan. He received his Ph. D.(1972) from Osaka University and subsequently was a member of the faculty of Pharmaceutical Sciences of the University. After two years (1975-1977) of postdoctoral work with Professor George Büchi at MIT, he moved back to Osaka University. He was promoted to Associate Professor in 1983 and to Full Professor of Osaka University in 1992. In 2008, he retired from Osaka University and joined Ritsumeikan University as the Dean of the Faculty of Pharmaceutical Sciences. From 2011 to 2015, he held Vice-President of the Research Organization of Science and Technology, Ritsumeikan University. Since April 2015, he has been Invited Research Professor and Director of Research Center for Drug Discovery and Pharmaceutical Development Science of the same University. He has a wide range of research interest in synthetic organic chemistry including the development of new asymmetric 
synthesis, new reagents, and the total synthesis of biologically active natural products. His current research interest is in hypervalent iodine chemistry.

He has published more than 500 original papers. His awards include the Pharmaceutical Society of Japan (PSJ) Award for Young Scientists (1986), the PSJ Award for Divisional Scientific Contribution (1997), the PSJ Award (2002), the Japanese Society for Process Chemistry (JSPC) Award for Excellence (2005), the Society of lodine Science (SIS) Award (2007), and the E.C. Taylor Senior Award (2017).

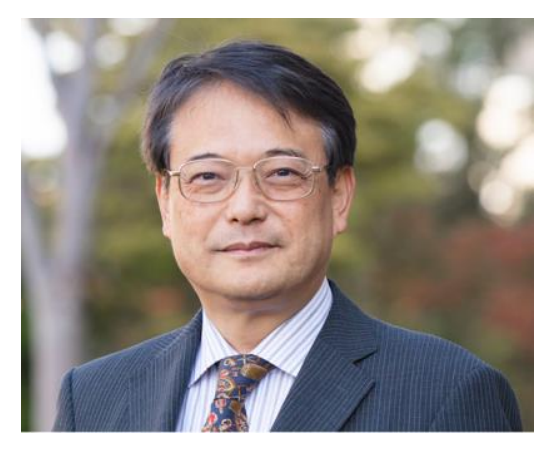

Tetsuya Kajimoto was born in 1960 and received M.S. in 1984 and Ph.D. in 1989 from Kyoto University. He was then appointed as Assistant Professor at Kumamoto University. After two years of postdoctoral study (1990-1991) with Professor Chi-Huey Wong at the Scripps Research Institute in San Diego (USA), he moved to the Frontier Research Program at the Institute of Physical and Chemical Research (RIKEN) in Japan (19911996). He was Associate Professor at Showa University (1996-1999), Tokyo University of Agriculture and Technology (1999-2003), and Kyoto Pharmaceutical University (2004-2007). Then, he was appointed as Professor at Suzuka University of Medical Science (2008) and Visiting Professor of Osaka University of Pharmaceutical Sciences (2009-2012). He moved to Ritsumeikan University as Professor at Institute of General Scientific Technology (2013-2016) and now is Professor at College of Pharmaceutical Sciences in Ritsumeikan University (2016-present). 